\title{
Impact of Hygiene on Malaria Transmission Dynamics: A Mathematical Model
}

Temidayo Oluwafemi* and Emmanuel Azuaba

Received : May 31, 2021
Abstract

Keywords: mathematical model, malaria, hygiene, stability analysis, basic reproduction number, lyapunov function, sensitivity analysis

\section{INTRODUCTION}

Malaria is one of the infectious diseases with an adverse effect on the human population. Some of the malaria parasites live in humans and the remaining is transmitted between human host and mosquito vector by the infected female Anopheles mosquitoes. In rare cases, people may be infected via contaminated blood, or a fetus may become infected by its mother during pregnancy or after delivery. Two of the five parasites species Plasmodium falciparum and Plasmodium vivax pose the greatest public health challenges [1] - [3] According to the World Health Organization [4], 219 million cases of malaria were reported in 89 countries and the estimated death cases were 435,000 with the African region carrying a disproportionately high share of the global malaria burden. Malaria is the third leading cause of death

Copyright Holder:

(C) Oluwafemi, T., and Azuaba, E. (2022)

First Publication Right:

Journal of Multidisciplinary Applied Natural Science

\section{Publisher's Note:}

Pandawa Institute stays neutral with regard to jurisdictional claims in published maps and institutional affiliations.

This Article is Licensed Under: most especially for children under five years, after pneumonia and diarrheal diseases.

The transmission dynamics of malaria mainly happened in poor environmental conditions. These conditions include unsafe water supplies, poor personal hygiene, poor sanitary facilities, poor living standards, and unhygienic food. Poor personal hygiene may result in water-borne diseases [5]. Poor environmental sanitation (hygiene) and housing conditions might be significant risk factors for malaria burden [6]. Enebeli et al. (2019) concluded that poor access to water, sanitation, and hygiene practices of caregivers directly relates to the prevalence of malaria among their children [7]. A mathematical model of malaria dynamics was developed with naturally acquired transient immunity in the presence of protected travellers [3]. A non-autonomous model was also developed to assess the impact of different microclimate conditions on the transmission dynamics of malaria [8]. A mathematical model has been proposed for the transmission dynamics of malaria by incorporating change via education as a control strategy [9]. The human population follows the susceptible-protected-exposed-infectious-recovered (SPEIR) pattern and the mosquito population follows susceptible-exposed-infectious (SEI) patterns. An analytical study was carried out to investigate the local stability of the system while the basic reproduction number was obtained using 
the next-generation matrix method. The result shows that the disease-free equilibrium of the system is locally asymptotically stable if $R_{0}<1$. The impact of temperature in malaria disease transmission dynamics was mathematically studied [10]. The SEIR model was suitable for the human population and LSEI compartment model was suitable for mosquito population. It was observed that temperature affects the transmission dynamics of malaria significantly. The impact of drugresistance in malaria transmission was also modelled [11]. Many mathematical models have been developed to study malaria dynamics but none has been discussed to study the impact of hygiene on malaria transmission dynamics as proposed in this work.

In this work, we propose a deterministic mathematical model for assessing the impact of hygiene on malaria transmission dynamics. The basic reproduction number is computed and the local and global stability of the disease-free equilibrium are established. Furthermore, the sensitivity analysis of the parameters is also evaluated.

\section{MATERIALS AND METHODS}

\subsection{Model Formulation}

In this model, the total human population denoted by $\left(N_{H}\right)$ is subdivided into unhygienic susceptible human population $\left(S_{u}\right)$, hygienic susceptible human population $\left(S_{h}\right)$, unhygienic infected human population $\left(I_{u}\right)$, hygienic infected human population $\left(I_{h}\right)$ and the recovered human population $\left(R_{h}\right)$. The mosquito population denoted by $\left(N_{v}\right)$ is subdivided into susceptible mosquitoes $\left(S_{v}\right)$ and infected mosquitoes $\left(I_{v}\right)$. See the equations 2.1 and 2.2.

$N_{H}=S_{u}+S_{h}+I_{u}+I_{h}+R$.

$N_{v}=S_{v}+I_{v}$

Let $\Lambda_{H}$ be the recruitment rate of the human population. A fraction $(1-\alpha) \Lambda_{H}$ enters unhygienic susceptible human class while the remaining fraction $\left(\alpha \Lambda_{H}\right)$ enters the hygienic susceptible human class. The unhygienic susceptible class is increased by the rate at which unhygienic human class lose immunity after recovery given as $\omega_{u}$, and reduced by the rate of progression to hygienic class $\left(\tau_{1}\right)$, the force of infection for the unhygienic class $\left(\lambda_{u}\right)$ and natural human death rate $\left(\mu_{H}\right)$. The hygienic susceptible human compartment is increased by the $\tau_{1}$, the rate at which hygienic human loss immunity after recovery at $\omega_{h}$, while the compartment is reduced by natural human death rate $\mu_{H}$ and the force of infection for the hygienic class $(1-\zeta) \lambda_{h}$. The $I_{u}$ is increased by $\lambda_{u}$ and reduced by $\mu_{H}$, rate of progression from $I_{u}$ to $I_{h}$ given as $\tau_{2}$. Malaria induced death for unhygienic human class and recovery for unhygienic human are denoted as $\delta_{u}$ and $\theta_{u}$. The $I_{h}$ is increased by $(1-\zeta) \lambda_{h}$ and $\tau_{2}$ then reduced by the recovery rate for a hygienic human class given as $\theta_{h}$. Malaria induced death for hygienic human class is denoted as $\delta_{h}$. The human recovery class $(R)$ is increased by $\theta_{h}$ and $\theta_{w}$ then reduced by $\mu_{H}, \omega_{h}$, and $\omega_{u}$. The $S_{v}$ is increased by the mosquito recruitment rate given as $\Lambda_{v}$, reduced by the mosquitoes death rate $\mu_{v}$, and force of infection for mosquito given as $\lambda_{v}$. Meanwhile, the $I_{v}$ is increased by $\lambda_{v}$ and $\mu_{v}$.

Given the above description and definitions of variables and parameters in Table 1 and 2, the following are the model equations:

$$
\begin{aligned}
\frac{d S_{u}}{d t} & =(1-\alpha) \Lambda_{H}-\left(\tau_{1}+\lambda_{u}+\mu_{H}\right) S_{u}+\omega_{u} R, \\
\frac{d S_{h}}{d t} & =\alpha \Lambda_{H}+\omega_{h} R+\tau_{1} S_{u}-\left((1-\zeta) \lambda_{h}+\mu_{H}\right) S_{h}, \\
\frac{d I_{u}}{d t} & =\lambda_{u} S_{u}-\left(\tau_{2}+\delta_{u}+\theta_{u}+\mu_{H}\right) I_{u}, \\
\frac{d I_{h}}{d t} & =(1-\zeta) \lambda_{h} S_{h}+\tau_{2} I_{u}-\left(\delta_{h}+\theta_{h}+\mu_{H}\right) I_{h}, \\
\frac{d R}{d t} & =\theta_{u} I_{u}+\theta_{h} I_{h}-\left(\omega_{u}+\omega_{h}+\mu_{H}\right) R, \\
\frac{d S_{v}}{d t} & =\Lambda_{v}-\lambda_{v} S_{v}-\mu_{v} S_{v}, \\
\frac{d I_{v}}{d t} & =\lambda_{v} S_{v}-\mu_{v} I_{v} \\
& \text { "where" } \\
\lambda_{u} & =\frac{b_{1} \beta_{v h} I_{v}}{N_{H}}, \lambda_{h}=\frac{b_{2} \beta_{v h} I_{v}}{N_{H}}, \quad b_{1}>b_{2}, \\
\lambda_{v} & =\frac{b_{3} \beta_{h v}\left(I_{u}+\rho I_{h}\right)}{N_{H}}, \delta_{u}>\delta_{h}, \theta_{h}>\theta_{u} .
\end{aligned}
$$




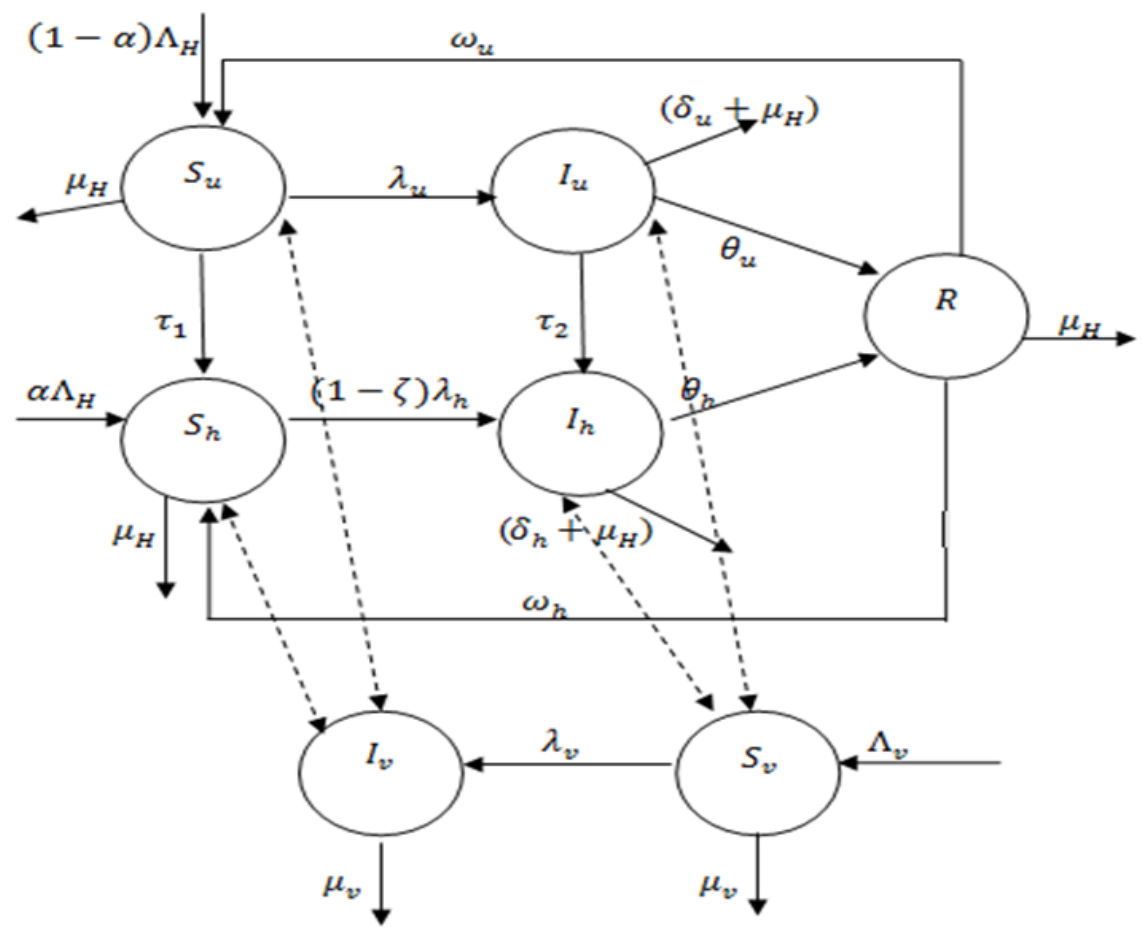

Figure 1. Model flow diagram.

\subsection{Invariant Region}

The invariant region can be obtained by the $t>0$ following theorem.

\section{Theorem 1}

The solutions of the model are feasible for all if they enter the invariant region

$$
\Omega=\Omega_{H} \times \Omega_{v}
$$

\section{Proof:}

Let

$$
\Omega=\left(S_{u}, S_{h}, I_{u}, I_{h}, R, S_{v}, I_{v}\right) \in \mathbb{R}_{+}^{7},
$$

be any solution of the system with non-negative initial conditions. Hence, all feasible solution set of the human population of the malaria model enters the region

$$
\begin{aligned}
\Omega_{H}= & \left\{\left(S_{u}, S_{h}, I_{u}, I_{h}, R\right) \in \mathbb{R}_{+}^{5}: S_{u} \geq 0, S_{h} \geq 0,\right. \\
& \left.I_{u} \geq 0, I_{h} \geq 0, R \geq 0, N_{H} \leq \frac{\Lambda_{H}}{\mu_{H}}\right\} .
\end{aligned}
$$

Similarly, the feasible solution set of the vector population enter the region

$$
\Omega_{v}=\left\{\left(S_{v}, I_{v}\right) \in \mathbb{R}_{+}^{2}: S_{v} \geq 0, I_{v} \geq 0, N_{v} \leq \frac{\Lambda_{v}}{\mu_{v}}\right\} .
$$

Therefore, the region $\Omega$ is positively invariant i.e. the solution remains positive for all initial values.

Thus, the model is biologically meaningful and mathematically well-posed in the domain $\Omega$.

\subsection{Disease Free Equilibrium (DFE)}

The DFE of the model equations can be found by setting the right hand of the model $(2.3)-(2.9)$ to zero, i.e.

$\frac{d S_{u}}{d t}=\frac{d S_{h}}{d t}=\frac{d I_{u}}{d t}=\frac{d I_{h}}{d t}=\frac{d R}{d t}=\frac{d S_{v}}{d t}=\frac{d I_{v}}{d t}=0$.

which gives

$(1-\alpha) \Lambda_{H}-\left(\tau_{1}+\lambda_{u}+\mu_{H}\right) S_{u}+\omega_{u} R=0$,

$\alpha \Lambda_{H}+\omega_{h} R+\tau_{1} S_{u}-\left((1-\zeta) \lambda_{h}+\mu_{H}\right) S_{h}=0$,

Table 1. Variables.

\begin{tabular}{cc}
\hline Symbols & Description \\
\hline$S_{u}$ & Unhygienic susceptible human \\
$S_{n}$ & Hygienic susceptible human \\
$I_{u}$ & Unhygienic infected human \\
$I_{n}$ & Hygienic infected human \\
$R$ & Recovered human \\
$S_{v}$ & Susceptible mosquitoes \\
$I_{v}$ & Infected mosquitoes \\
\hline
\end{tabular}


Table 2. Model Parameters.

\begin{tabular}{|c|c|}
\hline Symbols & Description \\
\hline$\Lambda_{H}$ & Recruitment rate of human population \\
\hline$\Lambda_{v}$ & Recruitment rate of mosquitoes \\
\hline$\tau_{1}$ & Progression from $S_{u}$ to $S_{n}$ \\
\hline$\tau_{2}$ & Progression from $I_{u}$ to $I_{n}$ \\
\hline$\delta_{u}$ & Disease - induced death for the unhygienic human class \\
\hline$\delta_{h}$ & Disease-induced death for the hygienic human class \\
\hline$b_{1}$ & Biting rate of mosquito for unhygienic human class \\
\hline$b_{2}$ & Biting rate of mosquito for hygienic human class \\
\hline$\beta_{v h}$ & Transmission probability of infection from mosquito to human \\
\hline$\beta_{h v}$ & Transmission probability of infection from human to mosquitoes \\
\hline$\lambda_{u}$ & The force of infection for unhygienic human class \\
\hline$\lambda_{h}$ & The force of infection for hygienic human class \\
\hline$\lambda_{v}$ & Force of infection for mosquitoes \\
\hline$b_{3}$ & Biting rate of mosquitoes \\
\hline$\zeta$ & Rate of reduction of infection for hygienic class \\
\hline$\rho$ & Modification parameter \\
\hline$\theta_{u}$ & Rate of recovery for unhygienic human class \\
\hline$\theta_{h}$ & Rate of recovery for hygienic human class \\
\hline$\omega$ & Rate at which recovered human become susceptible \\
\hline$\alpha$ & Hygienic rate \\
\hline$\mu_{H}$ & Natural human death rate \\
\hline$\mu_{v}$ & Natural death rate of mosquitoes \\
\hline$N_{H}$ & Total human population \\
\hline
\end{tabular}

At DFE, $I_{u}=I_{h}=I_{v}=0$,

So we have,

$$
(1-\alpha) \Lambda_{H}-\left(\tau_{1}+\mu_{H}\right) S_{u}=0
$$$$
\alpha \Lambda_{H}+\tau_{1} S_{u}-\mu_{H} S_{h}=0,
$$

$\Lambda_{v}-\mu_{v} S_{v}=0$. 
After computing simultaneously, we have

$S_{u}=\frac{(1-\alpha) \Lambda_{H}}{\left(\tau_{1}+\mu_{H}\right)}$,

$S_{h}=\frac{\Lambda_{H}\left(\tau_{1}+\alpha \mu_{H}\right)}{\mu_{H}\left(\tau_{1}+\mu_{H}\right)}$,

$S_{v}=\frac{\Lambda_{v}}{\mu_{v}}$.

Therefore, the DFE point of the model is given by

$E_{0}=\left(S_{u}^{0}, S_{h}^{0}, I_{u}^{0}, I_{h}^{0}, R^{0}, S_{v}^{0}, I_{v}^{0}\right)=\left(\frac{(1-\alpha) \Lambda_{H}}{\left(\tau_{1}+\mu_{H}\right)}, \frac{\Lambda_{H}\left(\tau_{1}+\alpha \mu_{H}\right)}{\mu_{H}\left(\tau_{1}+\mu_{H}\right)}, 0,0,0, \frac{\Lambda_{v}}{\mu_{v}}, 0\right)$.

\subsection{Basic Reproduction Number $\left(R_{0}\right)$}

The $R_{0}$ is defined as the number of secondary malaria infections produced by one infected individual in a completely susceptible community. The next-generation method [12] will be employed to compute $R_{0}$. The $F(x)$ is the rate of new infection appearance while $V(x)$ is the rate of transfer of individuals into compartments. Therefore,

$$
\begin{aligned}
& F=\left(\begin{array}{ccc}
0 & 0 & \frac{b_{1} \beta_{v h}(1-\alpha) \mu_{H}}{\left(\tau_{1}+\mu_{H}\right)} \\
0 & 0 & \frac{(1-\zeta) b_{2} \beta_{v h}\left(\alpha \mu_{H}+\tau_{1}\right)}{\left(\tau_{1}+\mu_{H}\right)} \\
\frac{b_{3} \beta_{h v} \Lambda_{\mathrm{V}} \mu_{\mathrm{H}}}{\Lambda_{\mathrm{H}} \mu_{\mathrm{V}}} & \frac{\rho b_{3} \beta_{h v} \Lambda_{\mathrm{V}} \mu_{\mathrm{H}}}{\Lambda_{\mathrm{H}} \mu_{\mathrm{V}}} & 0
\end{array}\right), \\
& V=\left(\begin{array}{ccc}
k_{1} & 0 & 0 \\
-\tau_{2} & k_{2} & 0 \\
0 & 0 & \mu_{v}
\end{array}\right),
\end{aligned}
$$

whereas

$$
k_{1}=\left(\tau_{2}+\delta_{u}+\theta_{u}+\mu_{H}\right), k_{2}=\left(\delta_{h}+\theta_{h}+\mu_{H}\right),
$$$$
k_{3}=\left(\omega+\mu_{H}\right) \text {. }
$$

$$
V^{-1}=\left(\begin{array}{ccc}
\frac{1}{k_{1}} & 0 & 0 \\
\frac{\tau_{2}}{k_{1} k_{2}} & \frac{1}{k_{2}} & 0 \\
0 & 0 & \frac{1}{\mu_{v}}
\end{array}\right),
$$$$
F V^{-1}=\left(\begin{array}{cc}
0 & 0 \\
0 & 0 \\
\frac{b_{3} \beta_{h v} \Lambda_{\mathrm{V}} \mu_{\mathrm{H}}}{\Lambda_{\mathrm{H}} \mu_{\mathrm{v}} k_{1}}+\frac{\tau_{2} \rho b_{3} \beta_{h v} \Lambda_{\mathrm{V}} \mu_{\mathrm{H}}}{\Lambda_{\mathrm{H}} \mu_{\mathrm{v}} k_{1} k_{2}} & \frac{\rho b_{3} \beta_{h v} \Lambda_{\mathrm{V}} \mu_{\mathrm{H}}}{\Lambda_{\mathrm{H}} \mu_{\mathrm{V}} k_{2}}
\end{array}\right.
$$$$
\left.\begin{array}{c}
\frac{b_{1} \beta_{v h}(1-\alpha) \mu_{H}}{\left(\tau_{1}+\mu_{H}\right) \mu_{v}} \\
\frac{(1-\zeta) b_{2} \beta_{v h}\left(\alpha \mu_{H}+\tau_{1}\right)}{\left(\tau_{1}+\mu_{H}\right) \mu_{v}} \\
0
\end{array}\right) \text {. }
$$

The $R_{0}$ is the largest eigenvalue or spectral radius of $F V^{1}$. Hence,

$$
R_{0}=\sqrt{\frac{b_{3} \beta_{v h} \beta_{h v} \Lambda_{v} \mu_{H}\left(b_{1} \mu_{H}(1-\alpha)\left(k_{2}+\tau_{2} \rho\right)+b_{2} k_{1} \rho\left(\alpha \mu_{H}+\tau_{1}\right)(1-\zeta)\right)}{\Lambda_{H} \mu_{v}^{2} k_{1} k_{2}\left(\tau_{1}+\mu_{H}\right)}} .
$$

\section{Theorem 2}

The DFE $E_{0}$ for model system is locally asymptotically stable if $R_{0}<1$ and unstable otherwise.

\section{Proof:}

At DFE, the Jacobian matrix is given by

$J=\left[\begin{array}{ccccccc}-\left(\tau_{1}+\mu_{H}\right) & 0 & 0 & 0 & \omega & 0 & 0 \\ \tau_{1} & -\mu_{H} & 0 & 0 & \omega & 0 & 0 \\ 0 & 0 & -k_{1} & 0 & 0 & 0 & 0 \\ 0 & 0 & 0 & -k_{2} & 0 & 0 & 0 \\ 0 & 0 & 0 & 0 & -k_{3} & 0 & 0 \\ 0 & 0 & 0 & 0 & 0 & -\mu_{v} & 0 \\ 0 & 0 & 0 & 0 & 0 & 0 & -\mu_{v}\end{array}\right]$

Table 3. Indices of Sensitivity.

\begin{tabular}{cc}
\hline Symbols & Sensitivity Index \\
\hline$\Lambda_{H}$ & -1 \\
$\Lambda_{v}$ & 1 \\
$\tau_{1}$ & -0.00013 \\
$\tau_{2}$ & -0.000041 \\
$\delta_{u}$ & -0.000041 \\
$\delta_{h}$ & -0.29 \\
$b_{1}$ & 0.00022 \\
$b_{2}$ & 1 \\
$\beta_{v h}$ & 1 \\
$\beta_{h v}$ & 1 \\
$b_{3}$ & 1 \\
$\zeta$ & -0.087 \\
$\rho$ & 1 \\
$\theta_{u}$ & -0.000015 \\
$\theta_{h}$ & -0.71 \\
$\alpha$ & -0.00011 \\
$\mu_{H}$ & 1 \\
$\mu_{v}$ & -2 \\
\hline
\end{tabular}




$J-\lambda I \mid$
$=\left[\begin{array}{ccccccc}-\left(\tau_{1}+\mu_{H}\right)-\lambda & 0 & 0 & 0 & \omega & 0 & 0 \\ \tau_{1} & -\mu_{H}-\lambda & 0 & 0 & \omega & 0 & 0 \\ 0 & 0 & -k_{1}-\lambda & 0 & 0 & 0 & 0 \\ 0 & 0 & 0 & -k_{2}-\lambda & 0 & 0 & 0 \\ 0 & 0 & 0 & 0 & -k_{3}-\lambda & 0 & 0 \\ 0 & 0 & 0 & 0 & 0 & -\mu_{v}-\lambda & 0 \\ 0 & 0 & 0 & 0 & 0 & 0 & -\mu_{v}-\lambda\end{array}\right]$,

The eigenvalues are:

$$
\begin{aligned}
& \lambda_{1}=-\left(\tau_{1}+\mu_{H}\right), \lambda_{2}=-\mu_{H}, \lambda_{3}=-k_{1}, \lambda_{4}=-k_{2}, \\
& \lambda_{5}=-k_{3}, \lambda_{6}=\lambda_{7}=-\mu_{v} .
\end{aligned}
$$

It is observed that all the eigenvalues are negative, this implies $R_{0}<1$ that at the DFE point is locally asymptotically stable, this means that malaria infection can be eliminated from the population.
2.5. Global stability of the Disease Free

Equilibrium (DFE)

\section{Theorem 3}

The DFE of the model system is globally asymptotically stable if $R_{0} \leq 1$.

\section{Proof:}

Consider the following Lyapunov function:

$$
\begin{aligned}
V(t)= & b_{3} \beta_{h v} \Lambda_{v} \mu_{H}\left(k_{2}+\tau_{2} \rho\right) I_{u}+b_{3} \rho \Lambda_{v} \mu_{H} \beta_{h v} k_{1} I_{h} \\
& +\Lambda_{H} k_{1} k_{2} \mu_{v} I_{v},
\end{aligned}
$$

Differentiating yield

\begin{tabular}{|c|c|c|}
\hline Symbols & Values & Source \\
\hline$\Lambda_{H}$ & 100 & [13] \\
\hline$\Lambda_{v}$ & 1000 & {$[14]$} \\
\hline$\tau_{1}$ & 0.25 & (Assumed) \\
\hline$\tau_{2}$ & 0.5 & (Assumed) \\
\hline$\delta_{u}$ & 0.13 & (Assumed) \\
\hline$\delta_{h}$ & 0.06 & (Assumed) \\
\hline$b_{1}$ & 0.17 & (Assumed) \\
\hline$b_{2}$ & 0.1 & (Assumed) \\
\hline$\beta_{v h}$ & 0.03 & [2] \\
\hline$\beta_{h v}$ & 0.09 & [2] \\
\hline$b_{3}$ & 0.12 & [15] \\
\hline$\zeta$ & 0.08 & (Assumed) \\
\hline$\rho$ & 0.5 & (Assumed) \\
\hline$\theta_{u}$ & 0.05 & (Assumed) \\
\hline$\theta_{h}$ & 0.15 & (Assumed) \\
\hline$\omega$ & 0.7902 & [14] \\
\hline$\alpha$ & 0.46 & (Assumed) \\
\hline$\mu_{H}$ & 0.00004 & [13] \\
\hline$\mu_{v}$ & 0.0000569 & [14] \\
\hline
\end{tabular}

$$
\begin{aligned}
\frac{d V}{d t}= & b_{3} \beta_{h v} \Lambda_{v} \mu_{H}\left(k_{2}+\tau_{2} \rho\right)\left(\lambda_{u} S_{u}-k_{1} I_{u}\right)+b_{3} \rho \Lambda_{v} \mu_{H} \beta_{h v} k_{1} \\
& \left((1-\zeta) \lambda_{h} S_{h}+\tau_{2} I_{u}-k_{2} I_{h}\right)+\Lambda_{H} k_{1} k_{2} \mu_{v}\left(\lambda_{v} S_{v}-\mu_{v} I_{v}\right),
\end{aligned}
$$

Table 4. Parameter values of model. 
At DFE, it was found that

$\begin{aligned} \frac{d V}{d t} \leq & \left(\frac{b_{3} \beta_{v h} \beta_{h v} \Lambda_{v} \mu_{H}\left(b_{1} \mu_{H}(1-\alpha)\left(k_{2}+\tau_{2} \rho\right)+b_{2} k_{1} \rho\left(\alpha \mu_{H}+\tau_{1}\right)(1-\zeta)\right)}{\left(\tau_{1}+\mu_{H}\right)}\right. \\ & \left.-\Lambda_{H} k_{1} k_{2} \mu_{v}^{2}\right) I_{v},\end{aligned}$

$\frac{d V}{d t} \leq\left(\frac{b_{3} \beta_{v h} \beta_{h v} \Lambda_{v} \mu_{H}\left(b_{1} \mu_{H}(1-\alpha)\left(k_{2}+\tau_{2} \rho\right)+b_{2} k_{1} \rho\left(\alpha \mu_{H}+\tau_{1}\right)(1-\zeta)\right)}{\Lambda_{H} k_{1} k_{2} \mu_{v}^{2}\left(\tau_{1}+\mu_{H}\right)}\right.$ -1) $I_{v}$

$\frac{d V}{d t} \leq\left(R_{0}^{2}-1\right) I_{v}$

From the equation above, $\frac{d V}{d t} \leq 0$, if $R_{0} \leq 1$. (2.24)

Hence, the DFE is globally asymptotically stable.

\subsection{Sensitivity Analysis}

In this section, sensitivity analysis is carried out to identify the parameters that have a great influence on the $R_{0}$. The sensitivity index of $R_{0}$ to a given parameter $\mathrm{P}$ is given by the relation

$\Pi_{P}^{R_{0}}=\frac{\partial R_{0}}{\partial P} \frac{P}{R_{0}^{\prime}}$

Table 3 shows the sensitivity indices of the basic reproduction number to the parameters. The parameters with positive indices indicate that the basic reproduction number increases as their values increase. While the parameters with negative sensitivity indices indicate an increase in these parameters will result in the decline of the basic reproduction number and vice-versa.

\section{RESULT AND DISCUSSIONS}

First, this system of model is biologically meaningful and mathematically well-posed in the given domain $\Omega$. The $R_{0}$ of the model is computed using the next-generation method. The existence of the disease-free equilibrium of the system is established and the condition for the local stability of the disease-free equilibrium and global stability of the disease-free equilibrium follows using the Lyapunov function. The DFE is locally asymptotically stable if $R_{0}<1$ and globally asymptotically stable if $R_{0} \leq 1$. Sensitivity analysis of the model equation is carried out as illustrated in Table 3. From the table, it shows that the natural death rate of mosquitoes $\left(\mu_{v}\right)$ is most sensitive to the Basic Reproduction Number.

\section{CONCLUSIONS}

In this work, the mathematical model to assess the impact of hygiene on malaria transmission dynamics was proposed and analyzed. The model is divided into the human population and vector (mosquito) population, the human population is further subdivided into the susceptible unhygienic human population, susceptible hygienic human population, infected unhygienic human population, infected hygienic human population and recovered human, while the vector population is subdivided into the susceptible vector and infected vector. We proved that the model equation is biologically meaningful and mathematically well-posed. The disease-free equilibrium (DFE) is established and it was observed that DFE is locally asymptotically stable if $R_{0}<1$ while globally asymptotically stable if $R_{0} \leq 1$ using Lyapunov function. Sensitivity analysis of the model parameters is carried out and it shows that the natural death rate of mosquitoes is most sensitive to the Basic reproduction Number.

This implies that individuals must continue to engage in activities that promote both personal hygiene and environmental hygiene so as reduce the growth of mosquito hence curbing the spread of malaria also government and other NonGovernmental Organizations (NGOs) must continue to intensify campaigns on hygienic practices at individual and community levels. Future studies can be carried out on this model such as: establishing and proving the existence of the unique endemic equilibrium point, analyzing the stability (local and global stability) of the endemic equilibrium point, and solving the model equations using any analytic method available.

\section{AUTHOR INFORMATION}

\section{Corresponding Author}

Temidayo Oluwafemi - General Studies Department, Newgate College of Health Technology, Minna - 920211 (Nigeria); Email: dayofemi985@gmail.com 
Author

Emmanuel Azuaba - Department of Mathematics, Bingham University Karu, Nassarawa - 962101 (Nigeria) ;

\section{REFERENCES}

[1] E. Azuaba, J. M. Orverem, Y. M. Kura and U. J. Dahiru. (2020). "Mathematical Approach for Malaria Disease in the Presence of Drug Therapy and Treatment". International Journal of Mathematics, and Its Applications. 8 (1): 77-88.

[2] S. Olaniyi, K. O. Okosun and S. O. Adesanya. (2018). "Global Stability and Optimal Control Analysis of Malaria Dynamics in the Presence of Human Travelers". The Open Infectious Diseases Journal. 10: 166-186. 10.2174/1874279301810010166.

[3] S. Olaniyi, K. O. Okosun, S. O. Adesanya and R. S. Lebelo. (2020). "Modeling Malaria Dynamics with Partial Immunity and protected travelers: optimal control and costeffectiveness analysis". Journal of Biological Dynamics. $14 \quad$ (1): 90-115. 10.1080/17513758.2020.1722265.

[4] F. Kogan. (2020). "Remote Sensing for Malaria (Monitoring and Predicting Malaria from Operational Satellites)". Springer, Cham. 10.1007/978-3-030-46020-4.

[5] G. O Mauti. E. M. Mauti and K. D. Kowanga. (2015). "Evaluation of Malaria Spread in Relation to Poor Environmental Conditions at Kibaha District (Tanzania)". Journal of Scientific \& Innovative Research. 4 (5) :203-206.

[6] T. Nkuo-Akenji, N. N. Ntonifor, M. B. Ndukum, E. L. Abongwa, A. Nkwescheu, D. N. Anong, M. Songmbe, M. G. Boyo, K. N. Ndamukong, and V. P. K. Titanji. (2006). "Environmental factors affecting malaria parasite prevalence in rural Bolifamba, South West Cameroon". African Journal of Health Sciences. 13 (1-2): 40-46.

[7] U. U. Enebeli, A. N. Amadi, O. K. Iro, E. T. Oparaocha, E. A. Nwoke, S. N. O. Ibe, N. N. Oti, U. M. Chukwuocha, C. R. Nwufo, C. O. Amadi, and I. Esomonu. (2019). "Assessment of Water, Sanitation and Hygiene Practices and the Occurrence of Childhood Malaria in Abia State, Nigeria". Researchjournali's Journal of Public Health. 5 (6): 1-15.

[8] D. Okuonghae, and A. Nwankwo. (2019). "Mathematical Assessment of the Impact of Different Microclimate Conditions on Malaria Transmission Dynamics". Mathematical Biosciences and Engineering. 16 (3): 1414-1444.

[9] A. N. Goni, and S. Musa (2018). "Modeling the Effect of Education-Based Intervention in the control of Malaria". Science World Journal. 13 (4).

[10] G. Bhuju, G. R.Phaijoo, and D. B. Gurung. (2018). "Mathematical Study on Impact of Temperature in Malaria Disease Transmission Dynamics". Advances in Computer Sciences. 1 (2).

[11] O. K. Okosun, and O. D. Makinde. (2011). "Modeling the Impact of Drug Resistance in Malaria Transmission and Its Optimal Control Analysis". International Journal of Physical Sciences. 6: 6479-6487. 10.5897/ IJPS10.542.

[12] O. Diekmann, J. A. Hesterbeek, and M. G. Roberts. (2010). "Construction of NextGeneration Matrices for Compartmental Models in Epidemics". Journal of the Royal Society of Biology, Interface. 7 (47): 875885. 10.1098/rsif.2009.0386.

[13] M. O. Oluwatayo. (2019). "Mathematical Model of the Coinfection Dynamics of Malaria-Toxoplasmosis in the Tropics". Biometrical Letters. $56 \quad$ (2): 139-163. 10.2478/bile-2019-0013. 10.2478/bile-2019$\underline{0013}$.

[14] E. A. Bakare and C. R. Nwozo. (2017). "Bifurcation and Sensitivity Analysis of Malaria-Schistosomiasis Coinfection Model". International Journal of Applied Computational Mathematics. 10.1007/s40819 -017-0394-5.

[15] S. Olaniyi, and O. S. Obabiyi. (2013). "Mathematical Model for Malaria Transmission Dynamics in Human and Mosquito Populations with Nonlinear Forces of Infection". International Journal of Pure and Applied Mathematics. 88 (1): 125-56. 
J. Multidiscip. Appl. Nat. Sci.

10.12732/ijpam.v88i1.10. 